\title{
Adaptive Harmonic Analysis
}

Arnold Heemink, Bartele de Jong and Harrie Prins

UDC 551.466 .713

\begin{abstract}
Summary
In this paper we describe a new approach to the harmonic analysis of the tide. For a number of reasons the harmonic "constants" are not really constant but vary slowly in time. Therefore, we introduce a narrow-band noise process to model the time-varying behaviour of these harmonic parameters. Furthermore, since the measurements available are not perfect, we also introduce a, possibly time-varying, measurement noise process to model the errors associated with the measurement process. By employing a Kalman filter to estimate the harmonic parameters recursively, the estimates can be adapted contineously to changing conditions. The adaptive harmonic analysis can be used for the on-line prediction of the astronomical tide or, since the Kalman filter also produces the covariance of the estimation error, to gain quantitative insight into the resolution of tidal constituents.
\end{abstract}

\section{Anpassungsfähige harmonische Analyse (Zusammenfassung)}

In diesem Artikel beschreiben wir eine neue Methode zur harmonischen Analyse der Gezeiten. Aus verschiedenen Gründen sind die harmonischen Konstanten nicht wirklich konstant, sondern sie variieren mit der Zeit. Deshalb führen wir einen stochastischen Prozeß ein, um das zeitvariable Verhalten dieser harmonischen Parameter zu modellieren. Außerdem führen wir auch einen möglichst zeitvariablen stochastischen Prozeß bei den Messungen ein, um Meßfehler zu modellieren, weil die vorhandenen Messungen nicht perfekt sind. Bei der Anwendung eines Kalmanfilters zur rekursiven Beurteilung der harmonischen Parameter können diese Wertungen sich ändernden Bedingungen laufend angepaßt werden. Die angepaßte harmonische Analyse kann man für die Berechnung der astronomischen Gezeiten benutzen, oder, weil das Kalmanfilter auch die Kovarianz des Bewertungsfehlers liefert, um einen quantitativen Einblick in die Auflösung von Gezeitenkonstanten zu gewinnen.

\section{Introduction}

The harmonic analysis of the tide is well-known and is used frequently to predict the astronomical tide (Godin [1972]). However, there are still difficulties associated with the harmonic method. If shallow water effects become significant, the number of constituents required to describe the tide becomes very large (D ro n k e r s [1964]). Along the coast of The Netherlands often more than hundred constituents are taken in consideration. There are probably many constituents that are still unknown. As a result, the harmonic "constants" that are taken into account appear to be time-varying during a year. Furthermore, since in The Netherlands the conditions change continuously due to channel dredging, sedimentation, and the building of two storm surge barriers, the harmonic "constants" also vary slowly from year to year.

Another difficulty associated with the harmonic analysis is that the theoretical values of the nodal corrections introduced to take into account the tidal frequency of one cycle per 18.61 year, are not always accurate. Moreover, if the tide is influenced by non-tidal phenomena such as the meteorological conditions, some harmonic constituents cannot be determined accurately or have to be left out of consideration. These effects also result in harmonic "constants" that appear to be time-varying. 
Due to the difficulties just described, we introduce in this paper the adaptive harmonic analysis. Here, we consider the harmonic "constants" as narrow-band stochastic parameters that vary randomly in time. Furthermore, since the measurements available are not perfect, we also introduce a measurement noise process to model the errors associated with the measurement process. By employing a Kalman filter to estimate the parameters recursively, the estimates can be adapted continuously to changing conditions (J a z w in ski [1970], M a y b e ck [1979]). By implementing the approach on-line, the short term predictions of the astronomical tide can be improved significantly. Furthermore, studying the time-varying behaviour of the amplitude and phase of the harmonic constituents may increase our insight into the performance of the underlying traditional harmonic analysis.

Another advantage of the use of our approach lies in its stochastic nature. By employing the Kalman filter, the covariance of the estimation error is also determined. This increases the insight into the resolution of tidal constituents, involving the record length as well as the narrow-band noise process and the measurement errors.

Finally, by implementing a Kalman filter that also adapts the measurement accuracy to changing conditions, it becomes possible to take into account that the measurements taken during stormy periods are less accurate. As a result, meteorological effects hardly influence the Kalman filter estimates of the amplitude and the phase of the harmonic constituents. Moreover, using such an adaptive filter it is also possible to overweight the water-level observations taken at the times of high and low water, so as to improve the predictions of the astronomical high and low water.

Since the original work of K a $1 \mathrm{~m}$ a n [1960], Kalman filters have been successfully used in numerous applications. Most of these filters were developed for the determination of satellite orbits and for the navigation of submarines, aircraft, and spaceships. In the last decennium Kalman filter techniques have also gained acceptance in meteorology (Ghil et al. [1981]), oceanography (Miller [1986]), and in several areas of hydraulics and hydrology (Chao-lin Chiu [1978]). However, these identification techniques have seldom been applied to the prediction of the astronomical tide. The use of the Kalman filter to estimate harmonic "constants" was first suggested by Mosetti [1983]. We have generalized his ideas by introducing the narrow-band process as a model of a harmonic constituent. Furthermore, to improve the estimation procedure we developed a Kalman filter that is able to adapt the estimates of the harmonic parameters as well as the measurement accuracy. Unlike Mosetti suggested, we first determine by means of a traditional harmonic analysis the stable harmonic constants and then we use our approach to model and predict the residual water elevation.

In this paper we first discuss in section two the narrow-band process. In section three we model the water-level residuals as the sum of a number of narrow-band processes and derive a state space representation of this model. The Kalman filter procedure is developed in section four. Finally, we describe in section five a number of applications of our approach to illustrate its advantages compared to the traditional harmonic analysis of the tide.

\section{Narrow-band processes}

Consider a weakly stationary stochastic process $\eta(t)$ with zero mean described by (Cramer and Leadbetter [1967], Wong [1971], Yagl om [1962]):

$$
\eta(t)=\int_{-\infty}^{\infty} e^{i \omega t} \mathrm{~d} Z(\omega)
$$

where $Z(\omega)$ is a complex valued stochastic process with zero mean and with independent increments such that:

$$
E\{\mathrm{~d} Z(\omega) \overline{\mathrm{d} Z(\omega)}\}=S(\omega) \mathrm{d} \omega
$$

with $S(\omega)$ as the two-sided energy spectral density function of the process $\eta(t)$. 
Since $\eta(t)$ is a real process we can rewrite (1) as:

$$
\eta(t)=\int_{-\infty}^{\infty} \cos (\omega t) d V(\omega)+\int_{-\infty}^{\infty} \sin (\omega t) d W(\omega)
$$

where:

$$
\begin{gathered}
\mathrm{d} V(\omega)=[\mathrm{d} Z(\omega)-\mathrm{d} Z(-\omega)] / 2 \\
\mathrm{~d} W(\omega)=\mathrm{i}[\mathrm{d} Z(\omega)+\mathrm{d} Z(-\omega)] / 2
\end{gathered}
$$

are mutually independent processes, both real and with independent increments such that:

$$
E\{\mathrm{~d} V(\omega) \mathrm{d} V(\omega)\}=E\{\mathrm{~d} W(\omega) \mathrm{d} W(\omega)\}=S(\omega) \mathrm{d} \omega
$$

If $\eta(t)$ is a narrow-band process, the spectral density function has a form as shown in figure 1. Here $\Delta \omega \ll \omega_{\mathrm{m}}$. In this case equation (3) can be rewritten as follows:

$$
\begin{aligned}
\eta(t)= & \int_{-\infty}^{\infty} \cos \left(\left[\omega_{\mathrm{m}}+v\right] t\right) \mathrm{d} V(v)+\int_{-\infty}^{\infty} \sin \left(\left[\omega_{\mathrm{m}}+v\right] t\right) \mathrm{d} W(v) \\
= & \cos \left(\omega_{\mathrm{m}} t\right)\left[\int_{-\infty}^{\infty} \cos (v t) \mathrm{d} V(v)+\int_{-\infty}^{\infty} \sin (v t) \mathrm{d} W(v)\right]+ \\
& \sin \left(\omega_{\mathrm{m}} t\right)\left[\int_{-\infty}^{\infty}-\sin (v t) \mathrm{d} V(v)+\int_{-\infty}^{\infty} \cos (v t) \mathrm{d} W(v)\right] \\
\cong & A_{\mathrm{m}}(t) \cos \left(\omega_{\mathrm{m}} t\right)+B_{\mathrm{m}}(t) \sin \left(\omega_{\mathrm{m}} t\right)
\end{aligned}
$$

where the mutual independent stochastic processes:

$$
\begin{aligned}
& A_{\mathrm{m}}(t)=\int_{-\infty}^{\infty} \cos (v t) \mathrm{d} V(v)+\int_{-\infty}^{\infty} \sin (v t) \mathrm{d} W(v) \\
& B_{\mathrm{m}}(t)=\int_{-\infty}^{\infty}-\sin (v t) \mathrm{d} V(v)+\int_{-\infty}^{\infty} \cos (v t) \mathrm{d} W(v)
\end{aligned}
$$

vary slowly in time since their spectral density:

$$
S_{\mathrm{m}}(v)=S\left(\omega-\omega_{\mathrm{m}}\right)
$$

is concentrated near $v=0$. Apparently, a narrow-band process can be considered as a harmonic oscillation with a slowly varying amplitude:

$$
A(t)=\sqrt{\left(A_{\mathrm{m}}(t)^{2}+B_{\mathrm{m}}(t)^{2}\right)}
$$

and phase:

$$
\phi(t)=\arctan \left[B_{\mathrm{m}}(t) / A_{\mathrm{m}}(t)\right]
$$




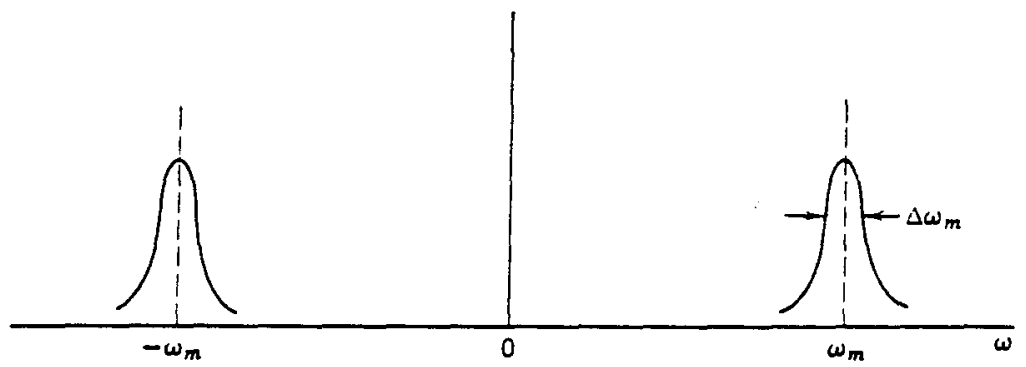

Fig. 1 Spectral density function of a narrow-band process

\section{State space representation}

In figure 2 the spectral density function of the residual water elevation at Vlissingen, located in the mouth of the Western Scheldt, is shown. The spectrum has been determined using hourly observations from 00:00, November 1, 1989 until 23:00, December 31, 1989. Using the harmonic constants of this measurement location, the astronomical tide has been eliminated as good as possible. In this figure we see both the meteorological effect at the low frequencies as well as still a number of narrow tidal bands in the spectrum. Apparently, the residuals $\zeta(t)$ can be considered as the sum of the metorological effect $m(t)$ and a number of narrow-band processes:

$$
\zeta(t) \cong \sum_{\mathrm{i}} \eta_{\mathrm{i}}(t)=\sum_{\mathrm{i}}\left[A_{\mathrm{i}}(t) \cos \left(\omega_{\mathrm{i}} t\right)+B_{i}(t) \sin \left(\omega_{\mathrm{i}} t\right)\right]+m(t)
$$

In order to derive a state-space model for $\zeta(t)$, we parameterise the spectral density functions $S_{\mathrm{i}}(v)$ of $A_{\mathrm{i}}(t)$ and $B_{\mathrm{i}}(t)$ according to:

$$
S_{\mathrm{i}}(v)=\frac{\sigma_{\mathrm{i}}^{2}}{a_{\mathrm{i}}^{2}+v^{2}}
$$

For small values of $a_{i}, S_{\mathrm{i}}(v)$ is a narrow-band spectrum (see figure 3 ). The autocovariance $r_{\mathrm{i}}(\tau)$ of $A_{\mathrm{i}}(t)$ and $B_{\mathrm{i}}(t)$ is given by (see figure 4 ):

$$
r_{\mathrm{i}}(\tau)=\int_{-\infty}^{\infty} \mathrm{e}^{\mathrm{i} v t} S(v) \mathrm{d} v=\frac{\sigma_{\mathrm{i}}^{2}}{2 a_{\mathrm{i}}} \mathrm{e}^{-a_{\mathrm{i}}|t|}
$$

The processes $A_{\mathrm{i}}(t)$ and $B_{\mathrm{i}}(t)$ can be modelled by means of the stochastic differential equations (J a z win ski [1970]):

$$
\begin{aligned}
& \mathrm{d} A_{\mathrm{i}}(t)=-a_{\mathrm{i}} A_{\mathrm{i}}(t) \mathrm{d} t+\sigma_{\mathrm{i}} \mathrm{d} \alpha(t) \\
& \mathrm{d} B_{\mathrm{i}}(t)=-a_{\mathrm{i}} B_{\mathrm{i}}(t) \mathrm{d} t+\sigma_{\mathrm{i}} \mathrm{d} \beta(t)
\end{aligned}
$$

where $\alpha(t)$ and $\beta(t)$ are mutually independent stochastic processes with zero mean and with independent increments such that:

$$
E\{\mathrm{~d} \alpha(t) \mathrm{d} \alpha(t)\}=E\{\mathrm{~d} \beta(t) \mathrm{d} \beta(t)\}=\mathrm{d} t
$$

Since $\sigma_{\mathrm{i}}$ is constant the stochastic differential equations (17) and (18) are the same in the Îto and Stratonowitz sense (J a z w in s k i [1970]). 
Using an Euler scheme, the stochastic differential equations (17) and (18) can be approximated by (Talay and Pardoux [1985]):

$$
\begin{aligned}
& \tilde{A}_{i}\left(t_{\mathrm{k}+1}\right)=\left(1-\Delta t a_{\mathrm{i}}\right) \tilde{A}_{\mathrm{i}}\left(t_{\mathrm{k}}\right)+\sigma_{\mathrm{i}} \Delta \alpha_{\mathrm{i}}\left(t_{\mathrm{k}}\right) \\
& \tilde{B}_{i}\left(t_{\mathrm{k}+1}\right)=\left(1-\Delta t a_{\mathrm{i}}\right) \widetilde{B}_{\mathrm{i}}\left(t_{\mathrm{k}}\right)+\sigma_{\mathrm{i}} \Delta \beta_{\mathrm{i}}\left(t_{\mathrm{k}}\right)
\end{aligned}
$$

Here $\tilde{A}_{\mathrm{i}}\left(t_{\mathrm{k}}\right)$ and $\tilde{B}_{\mathrm{i}}\left(t_{\mathrm{k}}\right)$ represent the numerical approximation of $A_{\mathrm{i}}\left(t_{\mathrm{k}}\right)$ and $B_{\mathrm{i}}\left(t_{\mathrm{k}}\right)$ respectively. Furthermore, $\Delta t$ is the time step and $\Delta \alpha_{\mathrm{i}}\left(t_{\mathrm{k}}\right)$ and $\Delta \beta_{\mathrm{i}}\left(t_{\mathrm{k}}\right)$ are mutually independent random variables with zero mean and variance $\Delta t$.

Defining the state vector $X\left(t_{\mathrm{k}}\right)$ :

$$
\boldsymbol{X}\left(t_{\mathrm{k}}\right)=\left[\tilde{A}_{1}\left(t_{\mathrm{k}}\right) \tilde{B}_{1}\left(t_{\mathrm{k}}\right) \quad \ldots \tilde{A}_{\mathrm{i}}\left(t_{\mathrm{k}}\right) \widetilde{B}_{\mathrm{i}}\left(t_{\mathrm{k}}\right) \ldots\right]^{T}
$$

the equation (20)-(21) can be rewritten in state space form:

$$
\boldsymbol{X}\left(t_{\mathrm{k}+1}\right)=\Phi \boldsymbol{X}\left(t_{\mathrm{k}}\right)+\Gamma \boldsymbol{W}\left(t_{\mathrm{k}}\right)
$$

where $\boldsymbol{W}\left(t_{\mathrm{k}}\right)$ consists of the random variables $\Delta \alpha_{i}\left(t_{\mathrm{k}}\right)$ and $\Delta \beta_{i}\left(t_{\mathrm{k}}\right)$. The covariance matrix $Q$ of $W\left(t_{\mathrm{k}}\right)$ can be shown to be $\mathrm{I} \Delta t . \Phi$ and $\Gamma$ are coefficient matrices that can be derived easily from the equations (20) and (21). relation:

If a measurement $\boldsymbol{Z}\left(t_{\mathrm{k}}\right)$ of the residual $\zeta\left(t_{\mathrm{k}}\right)$ is available, it can be modeled by the

$$
Z\left(t_{\mathrm{k}}\right)=\boldsymbol{M}\left(t_{\mathrm{k}}\right)^{T} \boldsymbol{X}\left(t_{\mathrm{k}}\right)+\boldsymbol{V}\left(t_{\mathrm{k}}\right)
$$

where $\boldsymbol{V}\left(t_{\mathrm{k}}\right)$ is the measurement noise with variance $\boldsymbol{R}\left(t_{\mathrm{k}}\right)$ that depends on the meteorological conditions. $\left.\boldsymbol{M ( t} t_{\mathrm{k}}\right)$ is the measurement vector:

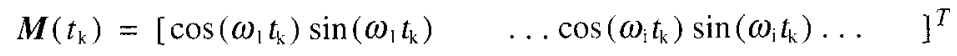

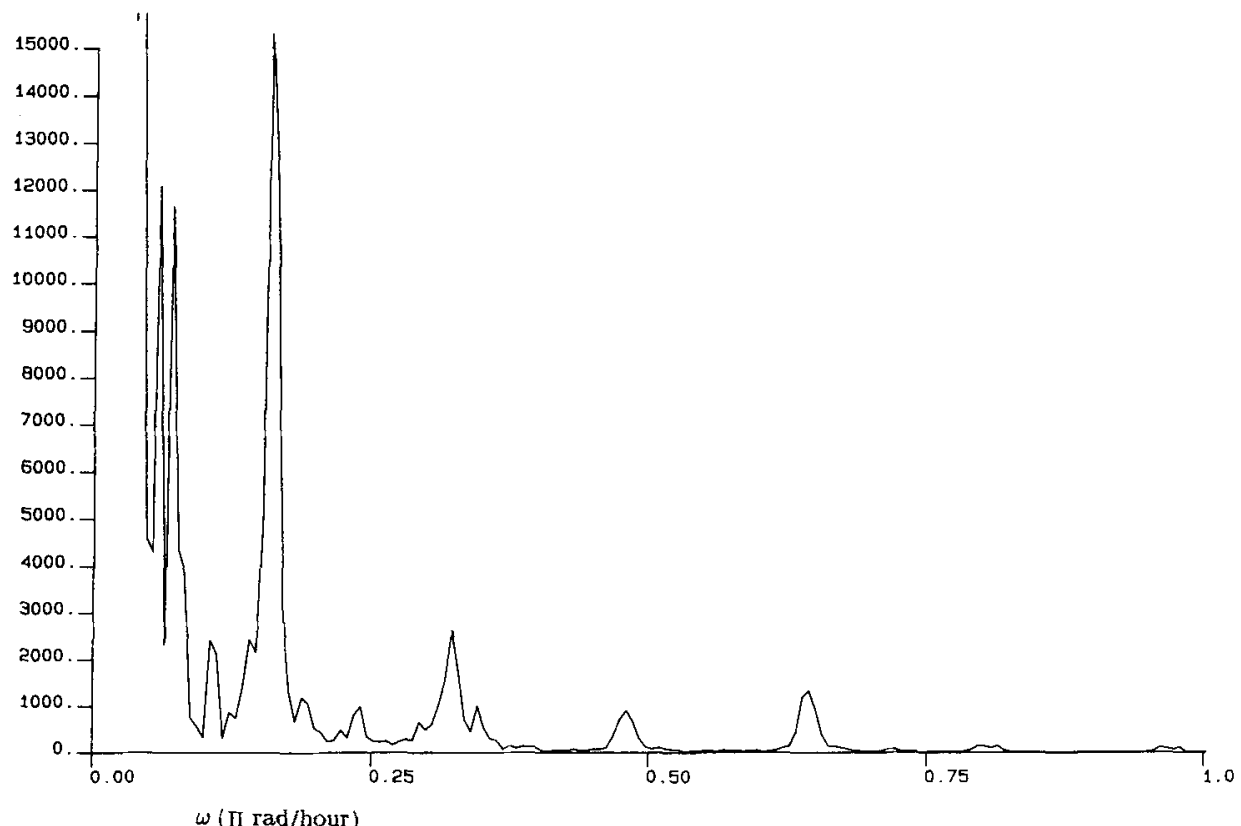

Fig. 2 Spectral density function of the residual tidal elevation 


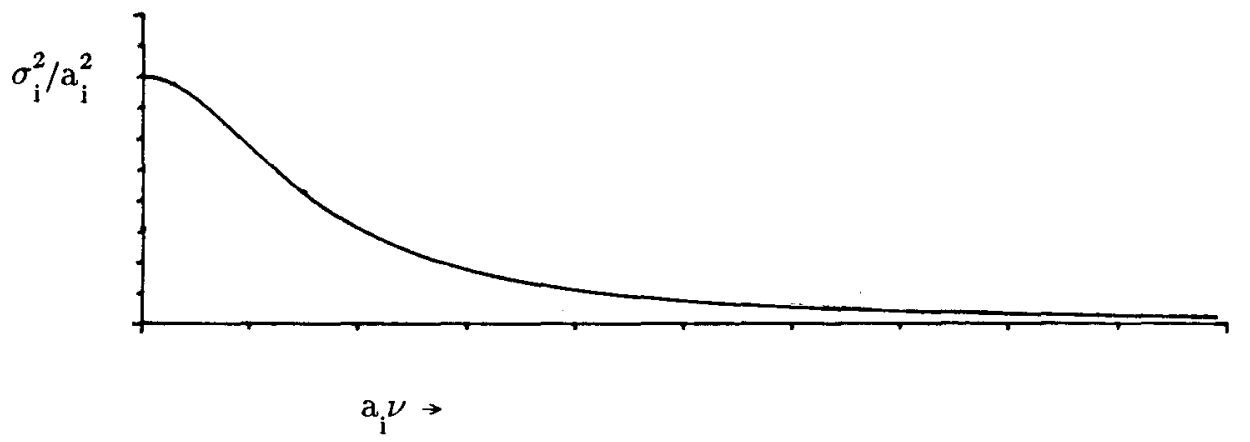

Fig. 3 Spectral density function $S_{\mathrm{i}}$
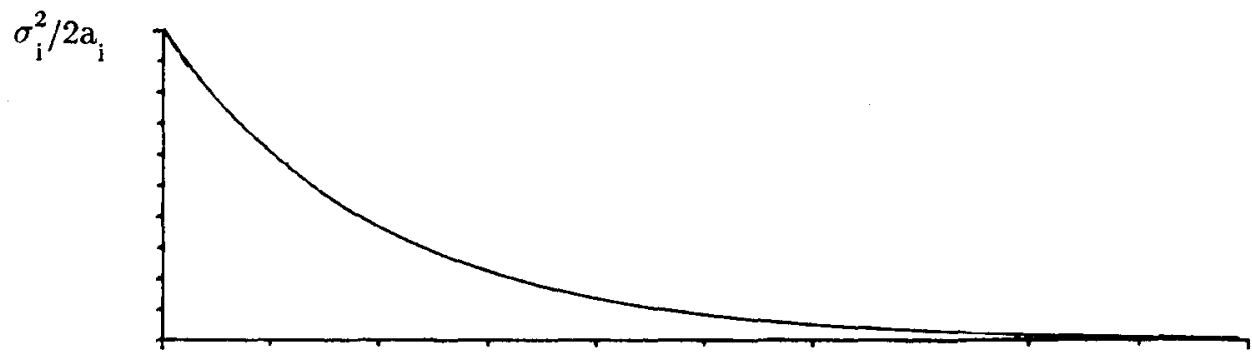

$\mathrm{t} / \mathrm{a}_{\mathbf{i}} \rightarrow$

Fig. 4 Auto covariance $r_{\mathrm{i}}$ of $S_{\mathrm{i}}$

\section{Kalman Filtering}

It is desired to combine the measurements taken from the actual system and modeled by the relation (24) with the information provided by the system model (23) in order to obtain an optimal estimate of the system state $\boldsymbol{X}\left(t_{\mathrm{k}}\right)$. If $\hat{\boldsymbol{X}}(\mathrm{k} / \mathrm{l})$ is defined as the least squares estimate of $\boldsymbol{X}\left(t_{\mathrm{k}}\right)$ based on the measurements $\boldsymbol{Z}\left(t_{1}\right), \boldsymbol{Z}\left(t_{2}\right), \ldots, \boldsymbol{Z}\left(t_{1}\right)$, and $\boldsymbol{P}(\mathrm{k} / \mathrm{l})$ represents the covariance matrix of the estimation error, recursive filter equations to obtain these quantities can be summarized as follows. The optimal state estimate is propagated from measurement time $t_{k-1}$ to measurement time $t_{k}$ by the equations:

$$
\begin{gathered}
\hat{\boldsymbol{X}}(k / k-1)=\boldsymbol{\Phi} \hat{\boldsymbol{X}}(k-1 / k-1) \\
\boldsymbol{P}(k / k-1)=\Phi \boldsymbol{P}(k-1 / k-1) \Phi^{\mathrm{T}}+\Gamma Q \Gamma^{\mathrm{T}}
\end{gathered}
$$

At measurement time $t_{\mathrm{k}}$, the new measurement $\boldsymbol{Z}\left(t_{\mathrm{k}}\right)$ becomes available. The estimate is updated by using the equations:

$$
\begin{gathered}
\hat{\boldsymbol{X}}(k / k)=\hat{\boldsymbol{X}}(k / k-1)+\boldsymbol{K}\left(t_{\mathrm{k}}\right)\left[\boldsymbol{Z}\left(t_{\mathrm{k}}\right)-\boldsymbol{M}\left(t_{\mathrm{k}}\right) \hat{\boldsymbol{X}}(k / k-1)\right] \\
\boldsymbol{P}(k / k)=\left[\mathrm{I}-\boldsymbol{K}\left(t_{\mathrm{k}}\right) \boldsymbol{M}\left(t_{\mathrm{k}}\right)\right] \boldsymbol{P}(k / k-1)
\end{gathered}
$$

where

$$
\boldsymbol{K}\left(t_{\mathrm{k}}\right)=\boldsymbol{P}(k / k-1) \boldsymbol{M}\left(t_{\mathrm{k}}\right)^{\mathrm{T}}\left[\boldsymbol{M}\left(t_{\mathrm{k}}\right) \boldsymbol{P}(k / k-1) \boldsymbol{M}\left(t_{\mathrm{k}}\right)^{\mathrm{T}}+\boldsymbol{R}\left(t_{\mathrm{k}}\right)\right]^{-1}
$$

is the filter gain. For the initial condition of the recursion we usually set $\hat{X}(0 / 0)=0$ and $P(0 / 0)$ very large to model that the initial estimate is not very accurate and has to be "forgotten" very soon by the filter. 
The filter just described is the celebrated Kalman filter for a discrete problem formulation. The derivation can be found, for instance, in M a y beck [1978]. The filter has a predictor-corrector structure. Based on all previous information, a prediction of the state of the system at time $t_{\mathrm{k}}$ is made by means of the equations (26) and (27). Once the prediction is known, it is possible to predict the next measurement by means of equation (24). When this measurement has become available, the difference between this measurement and its predicted value is used to update the prediction of the state of the system by means of equations (28) and (29).

The performance of the filter can be judged by monitoring the innovations $I\left(t_{\mathrm{k}}\right)$, defined as the difference between the measurements and the predictions of these measurements based on all previous information:

$$
\boldsymbol{I}\left(t_{\mathrm{k}}\right)=\boldsymbol{Z}\left(t_{\mathrm{k}}\right)-\boldsymbol{M}\left(t_{\mathrm{k}}\right)^{\mathrm{T}} \hat{\boldsymbol{X}}(k / k-1)
$$

It is easy to verify that:

$$
\begin{gathered}
\boldsymbol{E}\left\{I\left(t_{\mathrm{k}}\right)\right\}=0 \\
\boldsymbol{E}\left\{I\left(t_{\mathrm{k}}\right) I\left(t_{\mathrm{k}}\right)\right\}=\boldsymbol{M}\left(t_{\mathrm{k}}\right) \boldsymbol{P}(k / k-1) \boldsymbol{M}\left(t_{\mathrm{k}}\right)^{\mathrm{T}}+R\left(t_{\mathrm{k}}\right)
\end{gathered}
$$

Since the theoretical statistics of the innovations are known, the actual innovations can be monitored and compared with this description. By checking whether the innovations indeed possess their theoretical statistical properties we are able to judge whether the mathematical model satisfactorily describes the real system behaviour. Moreover, if the innovations do not possess their theoretical statistics, the variance of the measurement noise $R\left(t_{\mathrm{k}}\right)$ can be adapted ( $\mathrm{Jazwinski}$ [1970]). This feature is very useful since, as noted before, the measurement accuracy depends on the meteorological conditions. Furthermore, by using an adaptive filter it becomes possible to overweight the measurements taken at the times of high and low water, so as to improve the predictions of the astronomical high and low water.

Employing the Kalman filter, the covariance of the estimation error $P(k / k)$ can be used to gain insight into the resolution of tidal constituents involving both the record length as well as the measurement accuracy. Moreover, in case the measurement accuracy is not adapted to changing conditions, this error covariance does not depend on the actual data and can be precomputed.

The parameters $a_{i}$ and $\sigma_{i}$ have to be specified. Unfortunately, this knowledge is not very accurate. However, the filter performance turned out to be not very sensitve to the values of these parameters. In our case they were determined by comparing the parameterised spectral density $S_{i}(v)$ with observed spectra and by "trial and error", i. e. one employs the filter for various values of these parameters until one gets satisfactory filter performance. In fact, this is the calibration of the filter.

By choosing $Q \neq 0$ the harmonic parameters are expected to vary randomly in time. As a consequence, very old measurements will not be used by the filter to estimate the parameters. The memory of the filter, i. e. the "age" of the oldest measurement that is used by the filter, depends on the choise of $Q$. By increasing $Q$, the length of the memory will decrease.

Finally we note that for $a_{i}=0, \sigma_{i}=0$, and by taking $R=$ constant, a recursive least squares algorithm is obtained that produces, for the same problem formulation, the same results as the non-recursive least squares estimation algorithms generally used for the estimation of harmonic constants (God in [1972]).

\section{$5 \quad$ Results}

In this section we employ our approach to improve the on-line prediction of the astronomical tide at Vlissingen. Using the harmonic constants of this measurement location, the astronomical tide has been eliminated as good as possible to obtain the residual water elevation. Since in practice the meteorological effect is predicted on-line by means of storm surge forecasting models, we concentrate our attention on the prediction of the astronomical tide and 


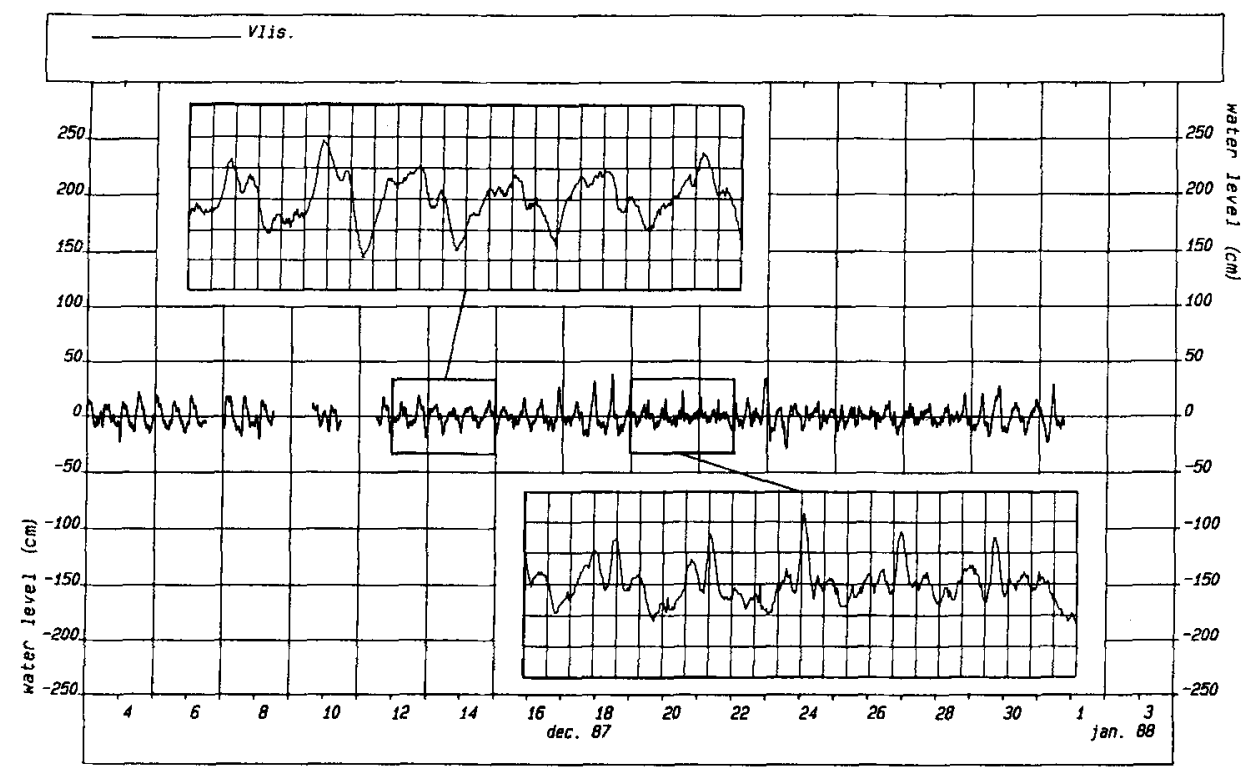

Fig. 5 Residuals in Vlissingen without the meteorological effects

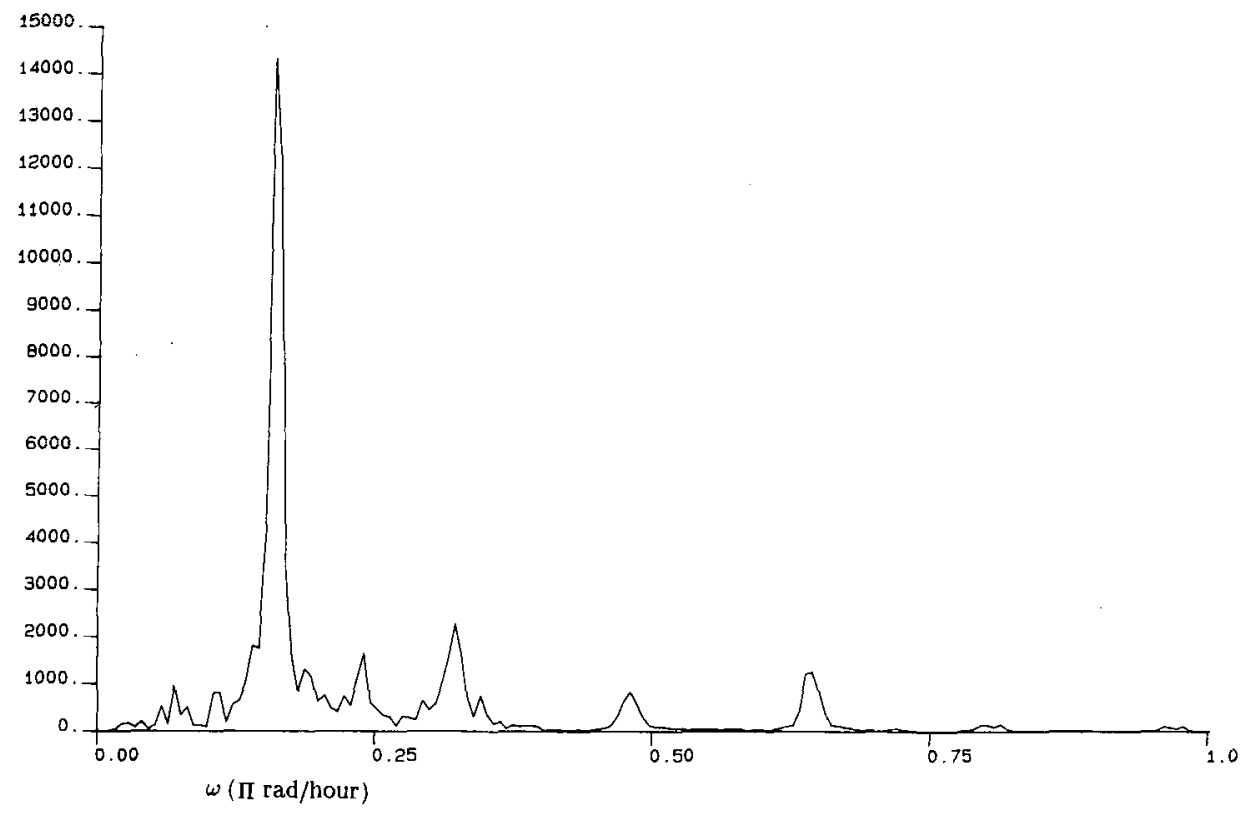

Fig. 6 Spectral density function of the residuals without the meteorological effects 
Lliminate the non-tidal effects from the residual water elevation by using a well-known low pass lilter (Godin [1972]). The resulting residuals from 00:00, December 1, 1989 until 23:00, December 31, 1989 are shown in figure 5. From this figure we clearly still see a periodic hehaviour, despite the fact that we have used more than hundred harmonic constituents to eliminate the astronomical tide. The spectral density function of the residuals in Vlissingen without the meteorological effect is shown in figure 6. It has been determined using hourly observations from 00:00, November 1, 1989 until 23:00, December 31, 1989. Again we see a number of narrow bands in the spectrum. The spectral density suggests the use of seven narrow-band processes with central frequencies $\omega_{1}, \omega_{2}, \omega_{3}, \omega_{4}, \omega_{5}, \omega_{6}$, and $\omega_{7}$ of the $M_{1}, M_{2}$, $M_{4}, M_{6}, M_{8}, M_{10}$, and $M_{12}$-tide respectively.

We first discuss the question of the resolution of the tidal constituents. As noted before, by employing the Kalman filter, the covariance of the estimation error can be used to gain insight into the resolution of tidal constituents involving both the record length as well as the measurement accuracy. Moreover, in case the measurement accuracy is not adapted to changing conditions, this error covariance does not depend on the actual data and can be precomputed. To illustrate this capability of the filter, we employ the approach to estimate the harmonic parameters and choose the standard deviation of the measurement noise $r=5 \mathrm{~cm}$. Let us first consider the deterministic case $a_{i}=0$ and $\sigma_{i}=0$. In figure 7 the standard deviation of the parameters $A_{2}$ and $B_{2}$ of the $M_{2}$-tide is shown as a function of time. Here, the measurements are available from 6:00, November 1, 1989. In this figure we also indicate the well-known Rayleigh criterion (Godin [1972]). From this figure we see that the harmonic parameters can be estimated accurately by using time series that are much shorter than the Rayleigh criterion indicates. Note also the oscillating behaviour of the standard deviation shown in figure 7 . This is caused by the measurement matrix $M\left(t_{\mathrm{k}}\right)$ introduced in equation (24). If $t_{\mathrm{k}}$ is such that:

$$
\sin \left(\omega_{2} t_{\mathrm{k}}\right) \approx 0
$$

the corresponding parameter $A_{2}$ cannot be estimated accurately from the measurements and, as a consequence, the standard deviation of the estimate of $A_{2}$ hardly decreases. Approximately three hours later we have:

$$
\cos \left(\omega_{2} t_{\mathrm{k}}\right) \approx 0
$$

and now the corresponding parameter $B_{2}$ cannot be estimated accurately.

In the figures 8 and 9 we have chosen respectively a very small and a very large measurement noise. In case the measurement noise is very small, the harmonic parameters can. be estimated extremly accurate using very short time series. However, for a very large measurement noise the record length that is required tends to be very long.

Now we introduce a system noise process with a covariance of the form $Q=q \mathrm{I}$. The standard deviation of the measurement noise was chosen to be $r=5 \mathrm{~cm}$. In the figures 10 and 11 again the standard deviation of the harmonic parameters $A_{2}$ and $B_{2}$ is shown as a function of time for different values of $q$. From figure 10 we see that a small system noise hardly effects the estimates of the parameters (compare with figure 7 where $q=0$ ). However choosing the system. noise very large, the memory of the filter becomes smaller than the record length that is required to separate the harmonic parameters. As a consequence the parameters cannot be estimated accurately.

Let us now employ the adaptive harmonic analysis to correct the prediction of the astronomical tide. Calibration of the filter results in values for the model parameters $a_{i}$ and $\sigma_{i}$ :

$$
\begin{gathered}
a_{i}=0.2510^{-4}, i=1, \ldots, 7 \\
\sigma_{1}=10^{-7} \\
\sigma_{i}=10^{-6}, i=2, \ldots, 7
\end{gathered}
$$

Here we note that the filter results are not very sensitive with respect to the choise of these parameters. 


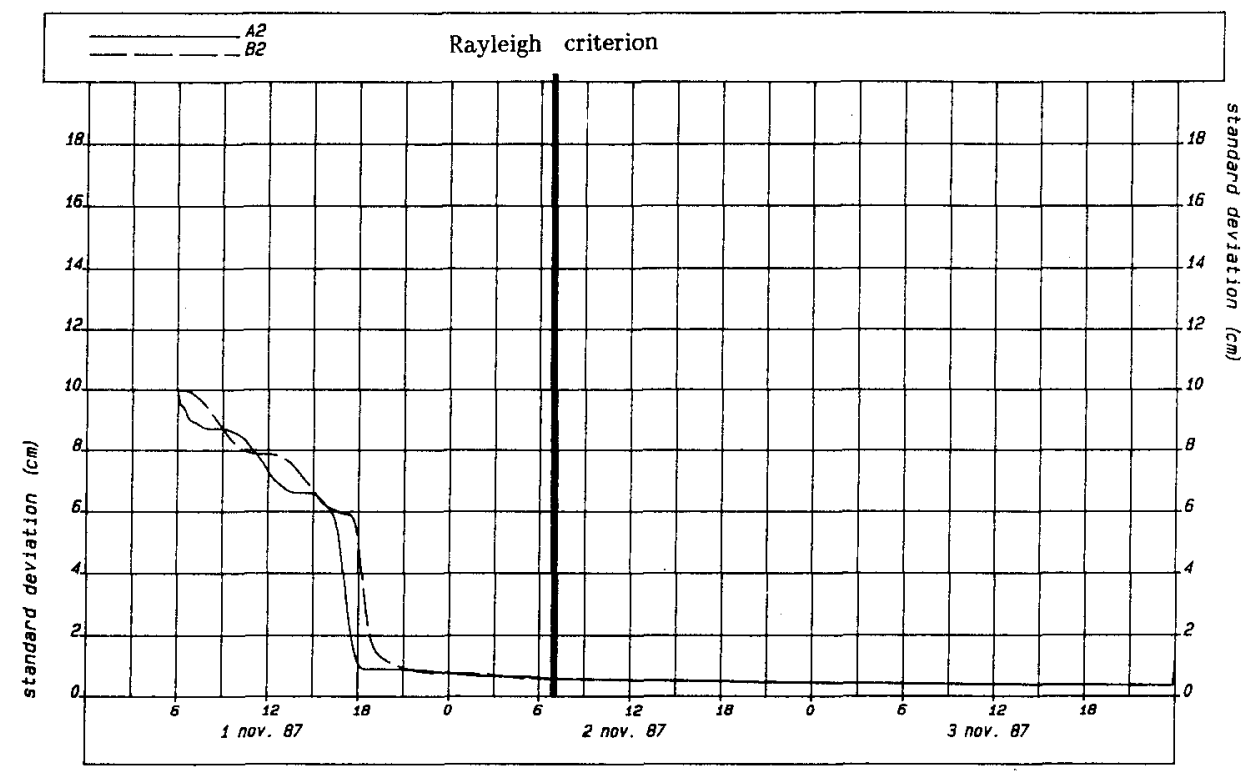

Fig. 7 Standard deviation of the components $A_{2}$ and $B_{2}$ in case $r=5 \mathrm{~cm}$ and $Q=0$

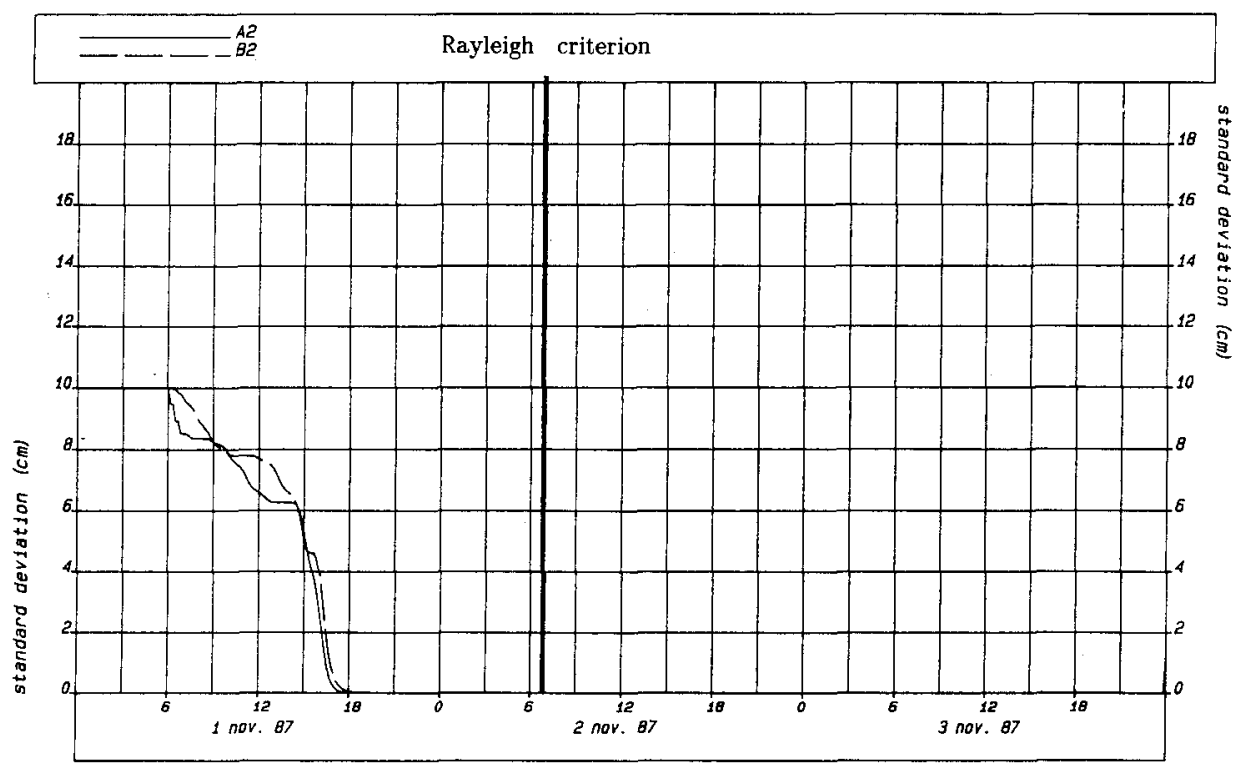

Fig. 8 Standard deviation of the components $A_{2}$ and $B_{2}$ in case $r=1 \mathrm{~cm}$ and $Q=0$ 


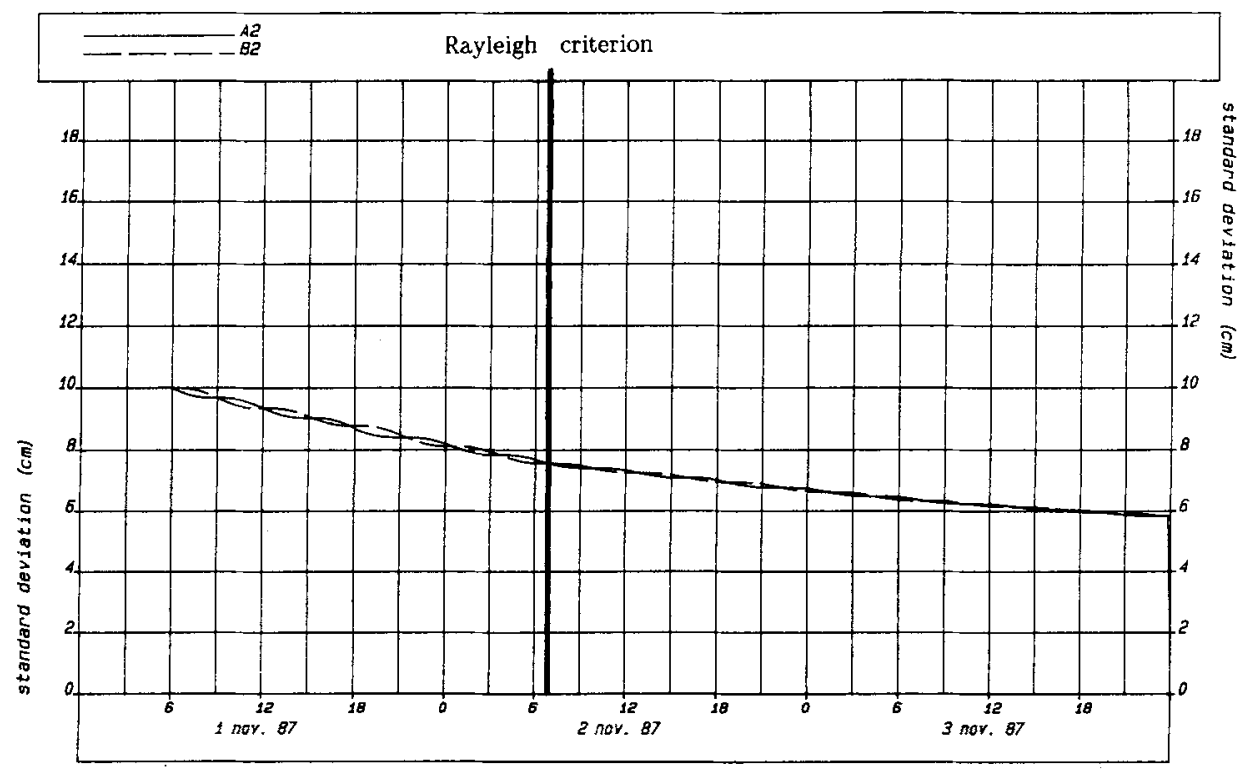

Fig. 9 Standard deviation of the components $A_{2}$ and $B_{2}$ in case $r=100 \mathrm{~cm}$ and $Q=0$

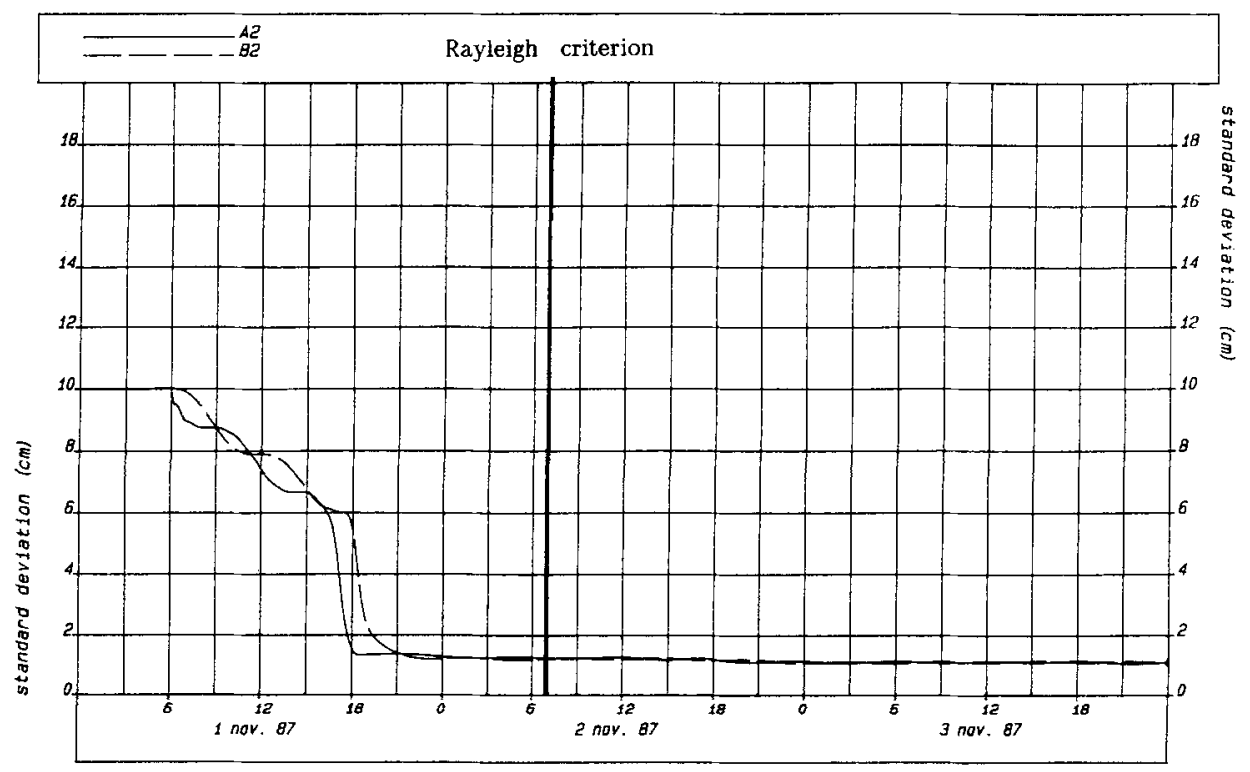

Fig. 10 Standard deviation of the components $A_{2}$ and $B_{2}$ in case $r=5 \mathrm{~cm}$ and $q=10^{-6}$ 


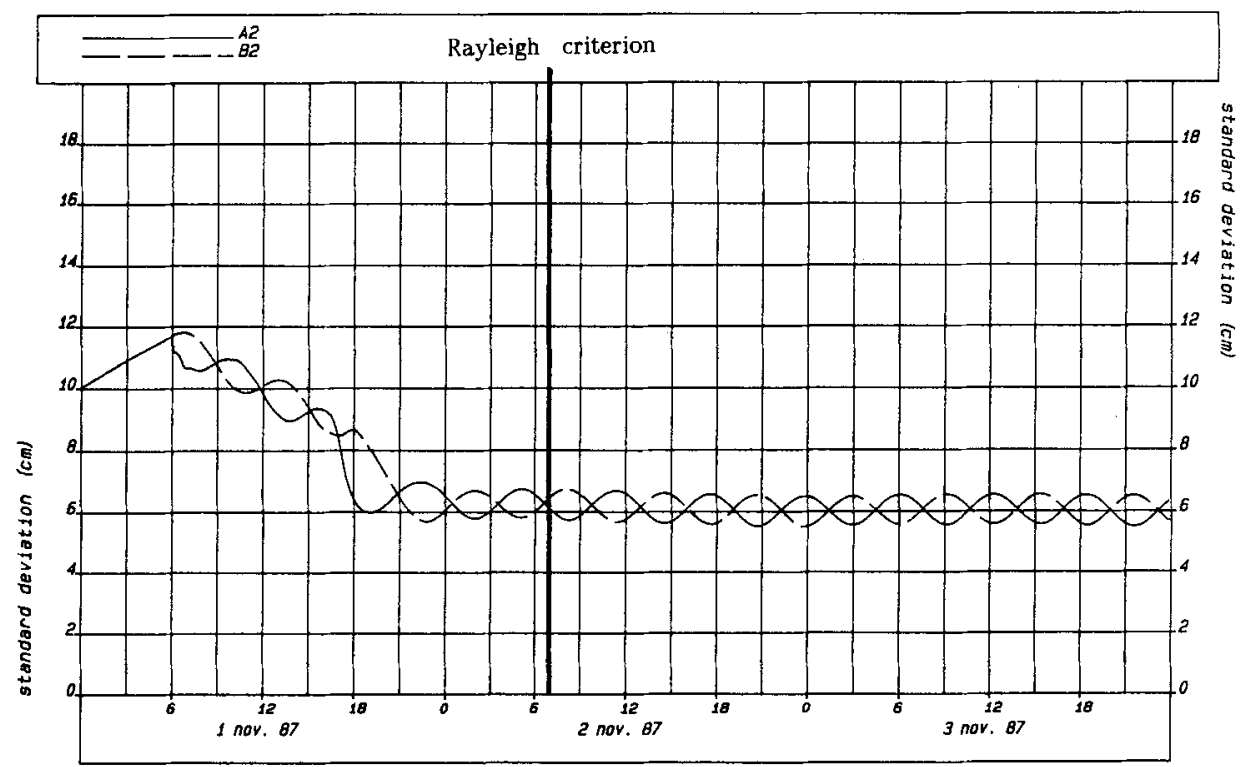

Fig. 11 Standard deviation of the components $A_{2}$ and $B_{2}$ in case $r=5 \mathrm{~cm}$ and $q=10^{-4}$

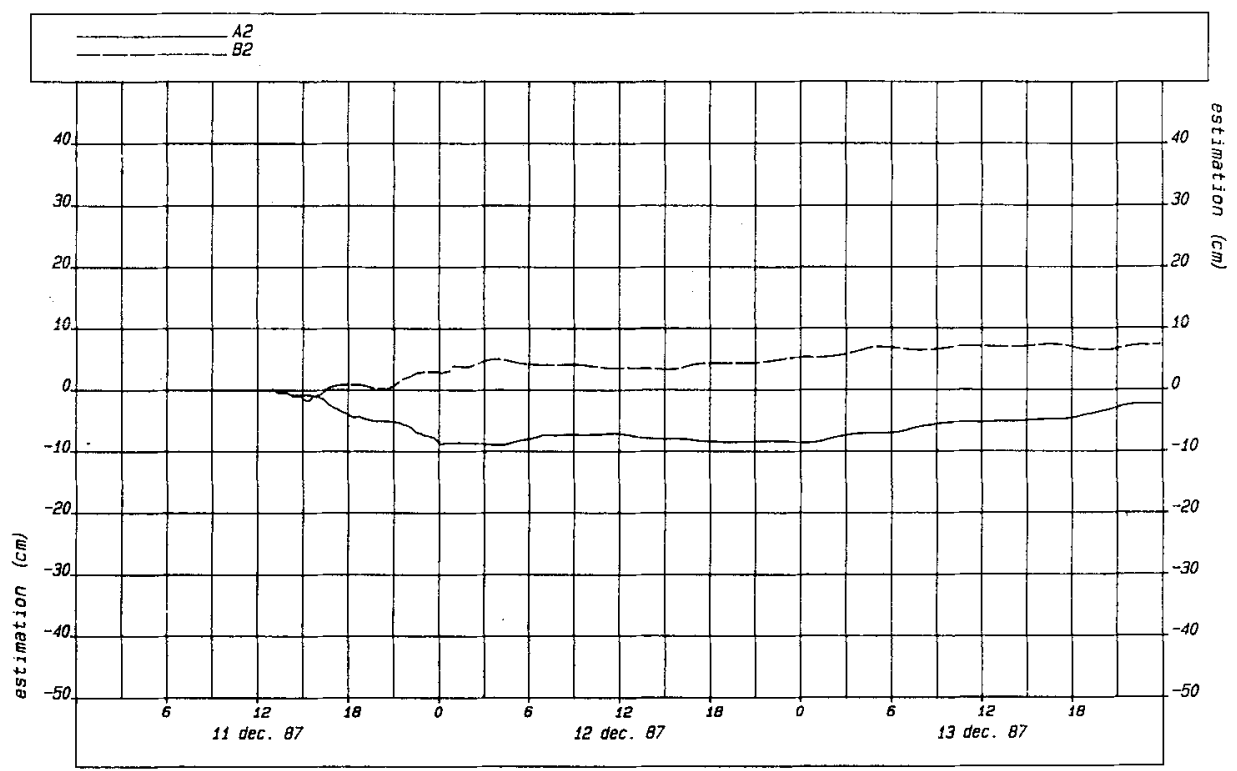

Fig. 12 Estimation of the components $A_{2}$ and $B_{2}$ 


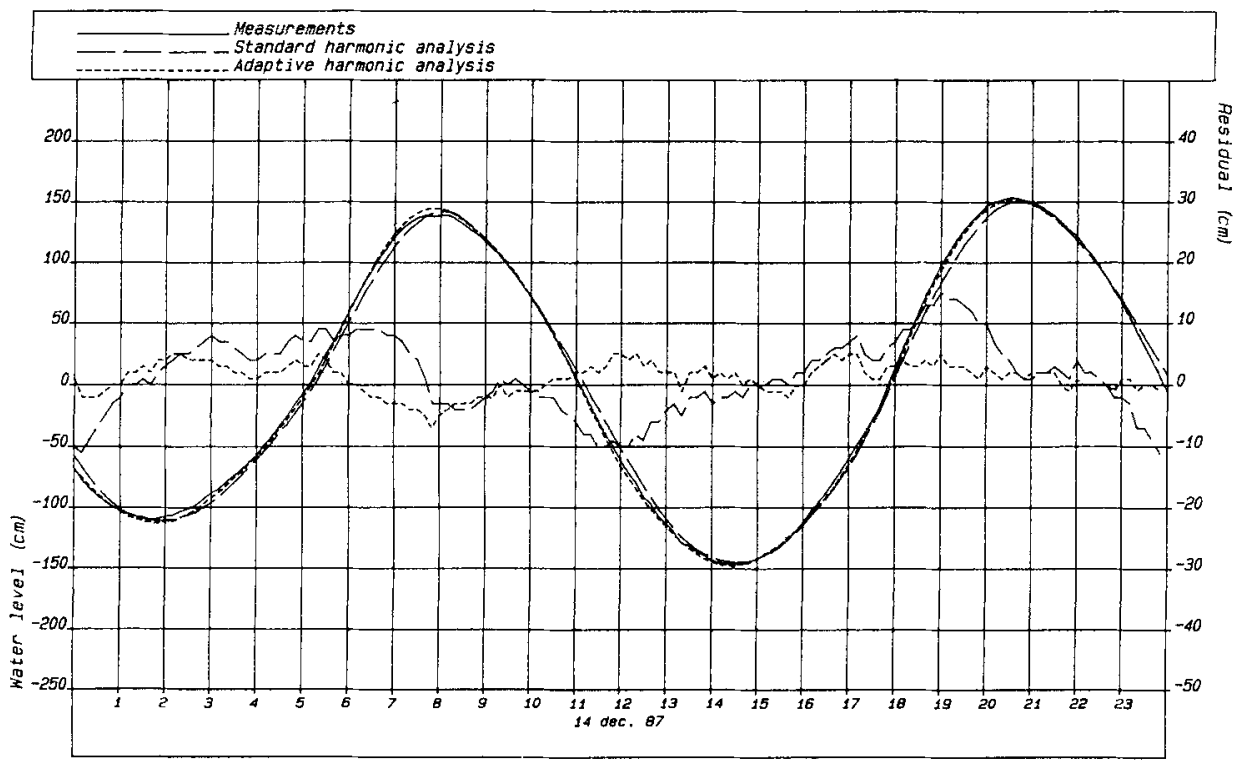

Fig. 13 Prediction of the standard harmonic analysis and the adaptive harmonic analysis

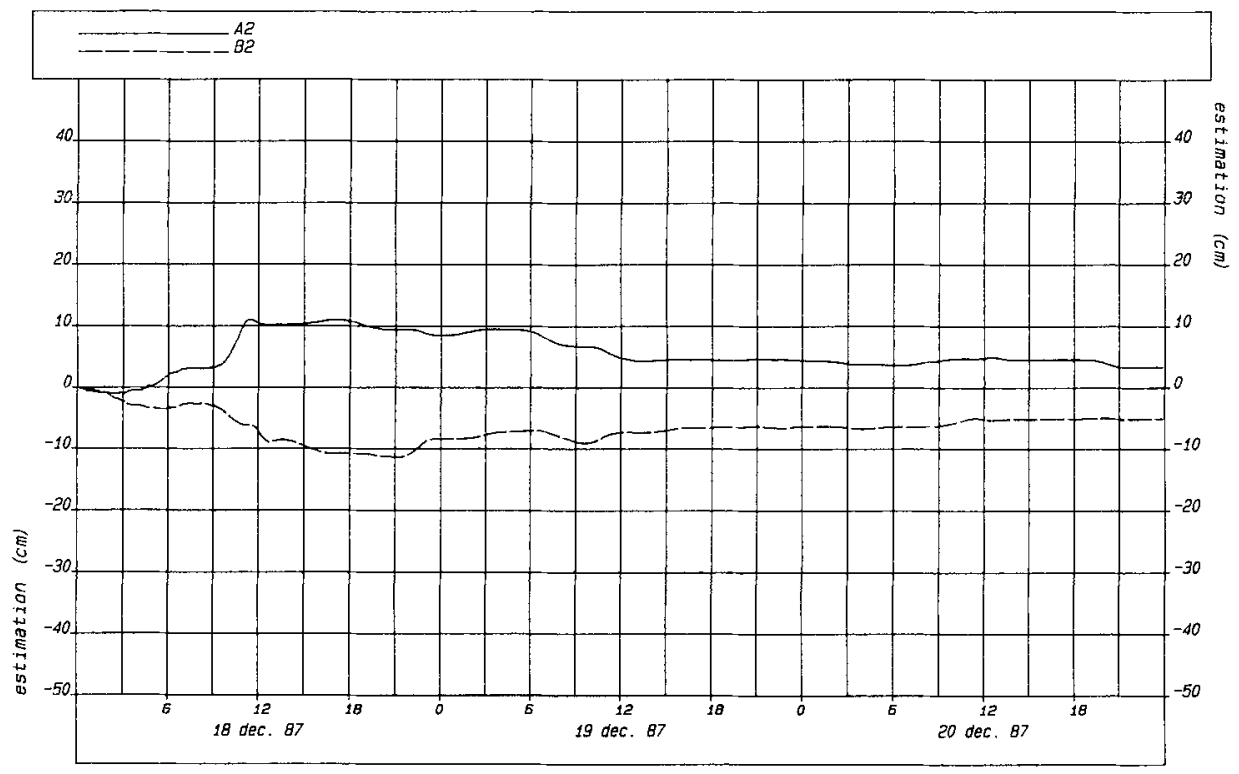

Fig. 14 Estimation of the components $A_{2}$ and $B_{2}$ 


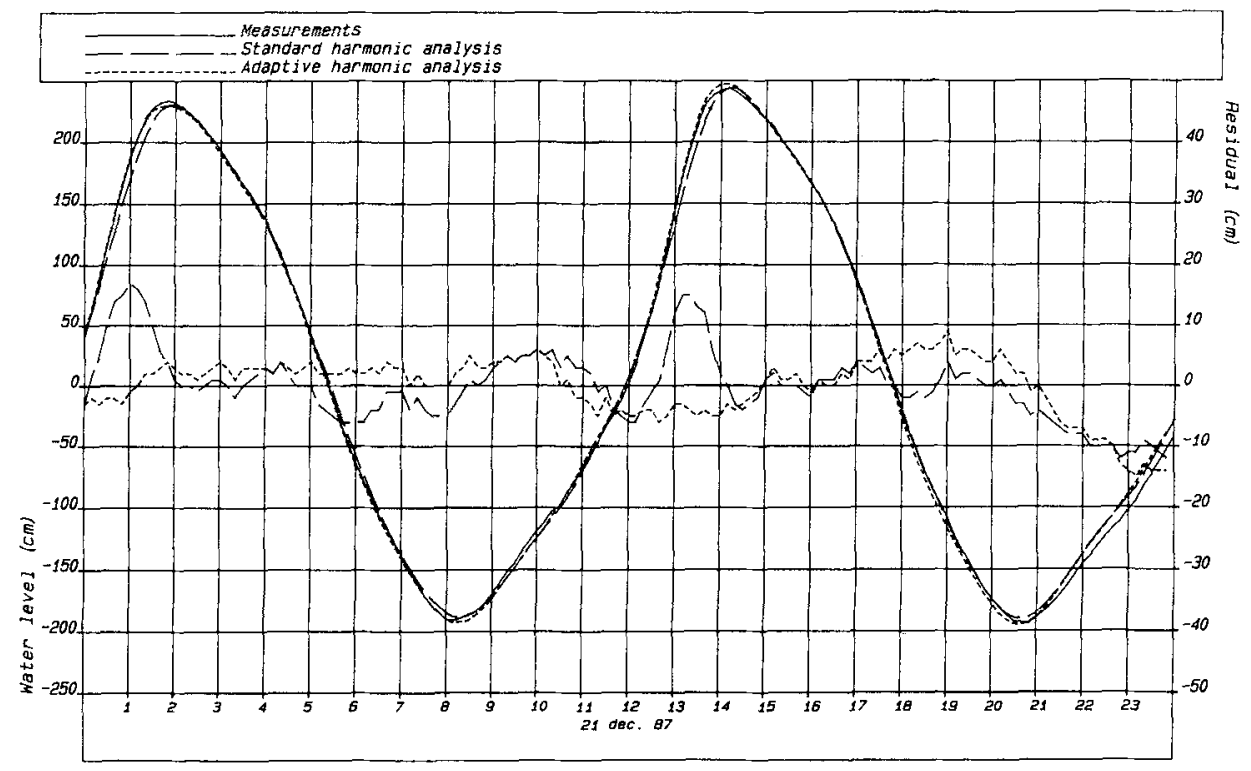

Fig. 15 Prediction of the standard harmonic analysis and the adaptive harmonic analysis

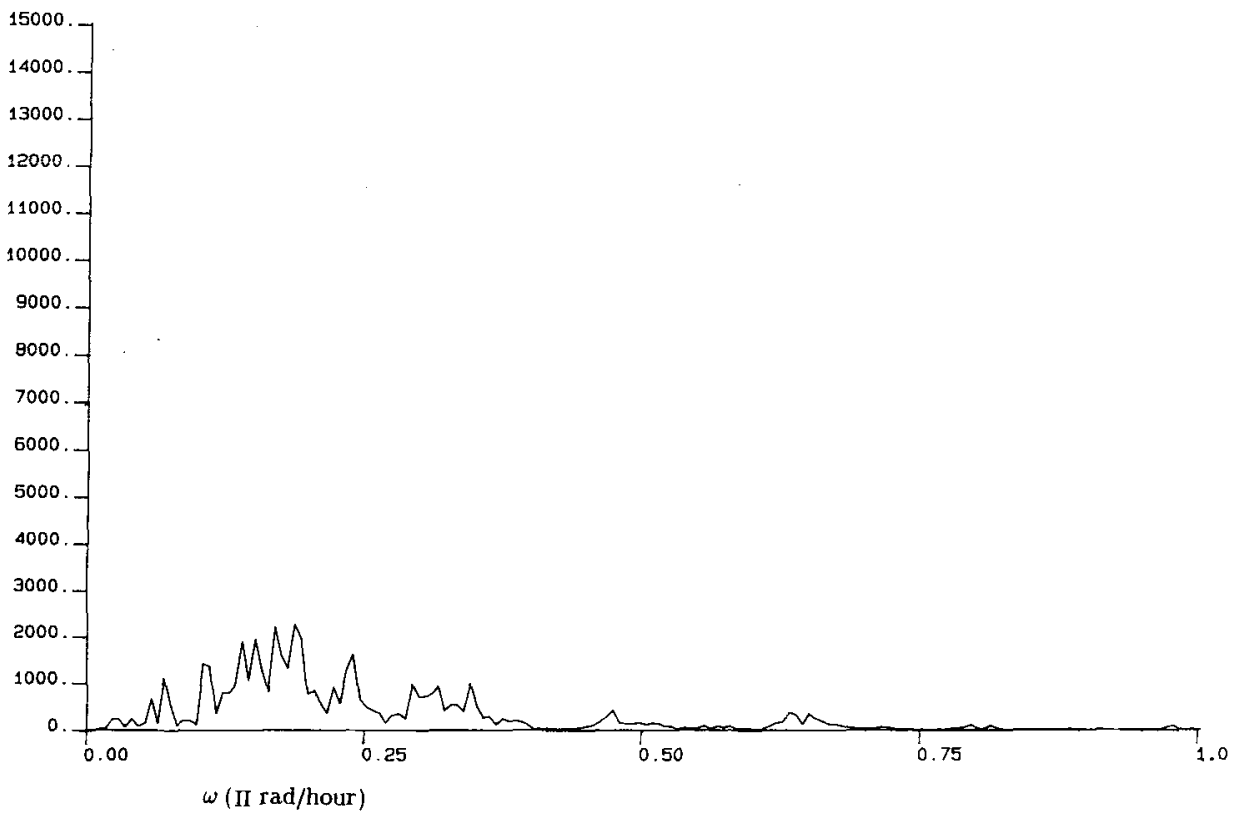

Fig. 16 Spectral density function of the errors of the adaptive analysis 
The filter is used on a routine basis to correct the prediction of the astronomical tide determined with the standard harmonic analysis. Here the estimates of the harmonic parameters are determined using the last 72 hourly measurements that are available. Based on these estimates, the correction of the astronomical tide is predicted for the next 24 hours. As example we show in the figures 12 and 13 respectively the estimation of the components $A_{2}$ and $B_{2}$ of the state from 00:00, December 11, 1989 until 23:00, December 13, 1989 and the filter prediction from 00:00 until 23:00 December 14, 1989. In figure 13 the predictions of the standard harmonic analysis are also shown. Similar results can be found in the the figures 14 and 15 . The approach has been used each day during the period from 00:00 November 1, 1989 until 23:00, December 31, 1989. In this period the variance of the prediction error of the standard harmonic analysis was reduced with approximately $40 \%$. In figure 16 we show the spectral density function of the prediction errors of the adaptive harmonic analysis. If we compare this sprectral density function with the spectral density function of the prediction errors of the standard harmonic analysis shown in figure 6, a significant improvement can be noticed.

\section{Conclusions}

In this paper we described the adaptive harmonic analysis of the tide. Here, we introduced a narrow-band process as a model of a harmonic constituent and employ Kalman fitering to estimate the time varying harmonic parameters recursively. The approach is used to improve the prediction of the astronomical tide. In this way the standard deviation of the prediction error can be reduced with approximately $40 \%$. Furthermore, since the Kalman filter also produces the covariance matrix of the estimation error, the approach can also be used to gain insight into the resolution of tidal constituents.

\section{Acknowledgements}

The authors gratefully acknowledge the contributions of Miss Regien Brouwer and the discussions with Mr. A. Langerak. 


\section{References}

Chao-1in Chiu (ed.), 1978: Application of Kalman Filter to Hydrology, Hydraulics and Water Resources. University of Pittsburg.

Cr a mer, H. and M. R. Le a d bet ter, 1967: Stationary and Related Stochastic Processes. John Wiley and Sons Inc.

Dronkers, J. J., 1964: Tidal Computations in Rivers and Coastal Waters. North Holland, Amsterdam.

Ghil, M., S. E. Cohn, J. Tavantzis, K. B ube, and E. Is a a c son, 1981: Application of Estimation Theory to Numerical Weather Prediction. In: Dynamic Meteorology: Data Assimilation Methods, Bengtsson, L., Ghil, M., and Källén, E. (eds.). New York: Springer, p. 139.

Godin, G., 1972: The Analysis of Tides. Toronto: University of Toronto Press.

Jazwinski, A. H., 1970: Stochastic Processes and Filtering Theory. New York: Academic Press.

Kalman, R. E., 1960: A New Approach to Linear Filter and Prediction Theory. J. Bas. Engng,, Vol. 82, p 17.

Maybeck, P. S., 1970: Stochastic Models, Estimation and Control. New York: Academic Press, Vol. 1.

Miller, R. N., 1986: Toward the Application of the Kalman-Bucy Filter to Regional Open Ocean Modelling. J. Physical Oceanography, Vol. 16, p 72.

Mos e t t i, R., 1983: A Kalman Filter Estimate of Tidal Harmonic Constants. Il Nuovo cimento C, Vol. 6, p 445.

Talay, D. and E. Pardoux, 1985: Discretisation and Simulation of Stochastic Differential Equations. Acta Appl. Math., Vol. 13, p 23.

W ong, E., 1971: Stochastic Processes in Information and Dynamical System. New York: Mc. Graw-Hill Book Company.

Y a g lo m, A. M., 1962: Theory of Stationary Random Functions. Englewood Cliffs, New Jersey: Prentice Hall Inc.

Eingegangen am 7. November 1990

Angenommen am 22. Januar 1991

Anschriften der Verfasser:

Prof. Dr. Ir. Arnold Heemink

Rijkswaterstaat, Tidal Waters Division,

P.O.B. 20907, 2500 EX The Hague, The Netherlands

Bartele de Jong and Harrie Prins

University of Twente, Department of Applied Mathematics

P.O.B. 217, 7500 AE Enschede, The Netherlands 